\title{
Evaluation of CMR predictors of right ventricular remodelling in dilated cardiomyopathy
}

\author{
Upasana Tayal ${ }^{1,2^{*}}$, Simon Newsome ${ }^{3}$, Ricardo Wage ${ }^{1}$, Aamir Ali ${ }^{1,2}$, Brian Halliday ${ }^{1}$, Zohreh Farzad ${ }^{1}$, \\ Dudley J Pennell', Stuart Cook ${ }^{2,4}$, Sanjay Prasad ${ }^{1,2}$ \\ From 19th Annual SCMR Scientific Sessions \\ Los Angeles, CA, USA. 27-30 January 2016
}

\section{Background}

We have previously identified that right ventricular systolic dysfunction (RVSD), present in one third of patients with non-ischaemic dilated cardiomyopathy (DCM), is an independent predictor of all cause mortality and cardiac transplantation. CMR provides a robust and reliable way of quantifying RVSD.

The natural history of RVSD in DCM has not been formally evaluated. We sought to evaluate whether baseline left ventricular systolic function is predictive of the development of RVSD and whether progression of right-sided ventricular impairment is linked to progression of leftsided ventricular impairment.

\section{Methods}

130 DCM patients (mean age 53.9 years, 65\% male) underwent 2 cardiovascular magnetic resonance (CMR) studies, with a median interval of 2.7 years between studies (IQR 1.4-4.7 years). CMR was performed on a 1.5T Siemens scanner.

DCM was diagnosed on conventional criteria (increased LV end diastolic volume indexed to body surface area and reduced left ventricular ejection fraction (LVEF) compared with reference ranges normalised for age and gender; absence of significant underlying coronary artery disease). RVSD was defined as right ventricular ejection fraction (RVEF) $<45 \%$.

Data are presented as mean \pm standard deviation. Correlation between interval change in RVEF and LVEF was assessed with Pearson's correlation test. ANCOVA was performed to identify independent predictors (Table 1) of the follow up RVEF adjusted for baseline RVEF. All statistical calculations were performed using R.

\section{Results}

Mean baseline LVEF was $42 \%( \pm 12 \%)$ and RVEF $53 \%$ $( \pm 16 \%)$. Thirty-two patients $(25 \%)$ had RVSD at baseline, and 22 at follow up (17\%). Mean follow up LVEF was $47 \%( \pm 13 \%)$ and RVEF $54 \%$ ( $\pm 14 \%)$.

When controlling for baseline RVEF, baseline LVEF was not predictive of follow up RVEF ( $\mathrm{p}=0.19$, 95\% confidence interval $-3 \%$ to $0.7 \%$ ). When controlling for potential confounders, baseline LVEF remained non significant in predicting follow up RVEF.

The interval change in RVEF was strongly correlated with the interval change in LVEF between CMR studies $(\mathrm{r}=0.6, \mathrm{p}=<0.0001$, figure 1$)$. Controlling for baseline RVEF, the interval change in LVEF between studies was strongly predictive of follow up RVEF. For every $10 \%$ increase in LVEF between studies, the follow up RVEF would be $4.3 \%$ higher ( $\mathrm{p}<0.0001,95 \%$ confidence interval $3.1 \%$ to $5.4 \%$ ). This remained highly significant even when adjusting for potential confounders (listed in Table 1).

\section{Conclusions}

These data show no evidence that progression of RVSD in DCM is dependent on baseline left ventricular systolic function.

However, adverse or reverse remodelling of RV function mirrors the change in LV function.

This suggests therefore that patients with significant LV impairment but normal RV function at baseline may not necessarily develop RVSD. However if LV function improves or deteriorates then RV function is likely to follow a similar course.

${ }^{1}$ Royal Brompton Hospital, London, United Kingdom

Full list of author information is available at the end of the article 
Table 1 Evaluated predictors of RV remodelling

\begin{tabular}{rr}
$\begin{array}{c}\text { CMR Imaging } \\
\text { Predictors }\end{array}$ & $\begin{array}{c}\text { Baseline LVEF, left ventricular mid wall fibrosis detected on LGE, mitral regurgitation, indexed left and right end diastolic and } \\
\text { end systolic volumes. }\end{array}$ \\
$\begin{array}{r}\text { Clinical Predictors } \\
\text { Age, gender, ethnicity, medication history (use of diuretic, beta blockers, ACE inhibitors, Aldosterone Antagonists), symptom } \\
\text { status (NYHA class), resting heart rate, and comorbidities (hypertension and atrial fibrillation). }\end{array}$ \\
\hline
\end{tabular}

Evaluated predictors of RV remodelling (LVEF/RVEF=left/right ventricular ejection fraction, LGE= late gadolinium enhancement, NYHA= New York Heart Association symptom classification)

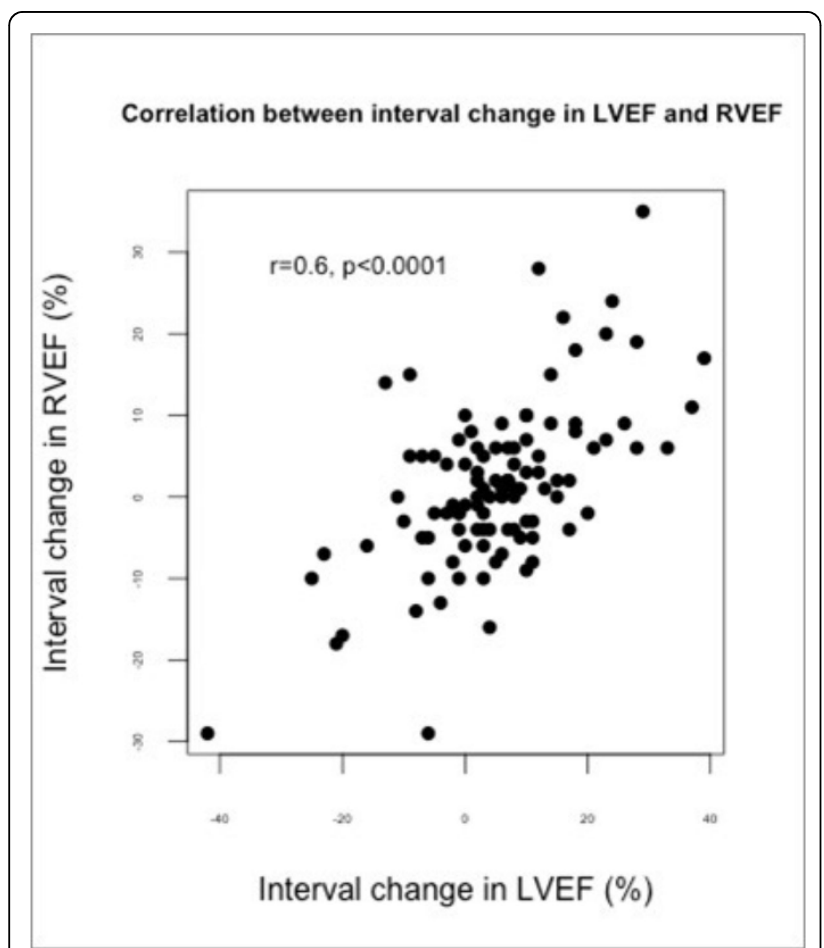

Figure 1 Scatterplot showing the correlation between the interval change in LVEF and RVEF between CMR studies. A positive interval change indicates reverse remodelling; a negative interval change indicates adverse remodelling.

\section{Authors' details}

${ }^{1}$ Royal Brompton Hospital, London, United Kingdom. ${ }^{2}$ Imperial College, London, United Kingdom. ${ }^{3}$ Department of Medical Statistics, London School of Hygiene and Tropical Medicine, London, United Kingdom. ${ }^{4}$ Duke National University Singapore, Singapore, Singapore.

Published: 27 January 2016

doi:10.1186/1532-429X-18-S1-P281

Cite this article as: Tayal et al.: Evaluation of CMR predictors of right ventricular remodelling in dilated cardiomyopathy. Journal of

Cardiovascular Magnetic Resonance 2016 18(Suppl 1):P281.

\section{Submit your next manuscript to BioMed Central} and take full advantage of:

- Convenient online submission

- Thorough peer review

- No space constraints or color figure charges

- Immediate publication on acceptance

- Inclusion in PubMed, CAS, Scopus and Google Scholar

- Research which is freely available for redistribution

Submit your manuscript at www.biomedcentral.com/submit 